\title{
Relationship between Low Vegetable Consumption, Increased High-Sensitive C-Reactive Protein Level, and Cardiometabolic Risk in Korean Adults with Tae-Eumin: A Cross-Sectional Study
}

\author{
Jieun Kim $\left(\mathbb{D}\right.$, Kyoungsik Jeong $\left(\mathbb{D}\right.$, Siwoo Lee $\mathbb{D}^{D}$, and Younghwa Baek \\ Future Medicine Division, Korea Institute of Oriental Medicine, Daejeon 34054, Republic of Korea \\ Correspondence should be addressed to Younghwa Baek; aori79@kiom.re.kr
}

Received 5 April 2021; Accepted 24 April 2021; Published 11 May 2021

Academic Editor: Swee Keong Yeap

Copyright (c) 2021 Jieun Kim et al. This is an open access article distributed under the Creative Commons Attribution License, which permits unrestricted use, distribution, and reproduction in any medium, provided the original work is properly cited.

\begin{abstract}
An anti-inflammatory diet has many beneficial effects on cardiometabolic diseases. Constitution type of traditional Korean medicine can predict cardiometabolic risk factors. We examined the relationship between vegetable consumption and the highsensitive C-reactive protein (hs-CRP) level on cardiometabolic risk factors in Korean adults by constitution types. Data from 1,983 eligible participants (mean age, 44.3 years) were included in the present cross-sectional study. The inflammatory status of the participants was categorized into low- $(<3.0 \mathrm{mg} / \mathrm{L})$ or high-risk $(\geq 3.0 \mathrm{mg} / \mathrm{L})$ groups based on their constitution types. Cardiometabolic risk factors (abdominal obesity, elevated triglycerides, reduced high-density lipoprotein-cholesterol, elevated blood pressure, elevated fasting plasma glucose, and $\geq 2$ concurrent cardiovascular diseases (CVDs) risk factors) and dietary assessment of the participants were assessed. A total of $11.1 \%$ of Tae-eumin (TE) and $4.9 \%$ of non-TE groups had a higher hs-CRP level (TE: 6.6 \pm 0.2 , non-TE: $8.4 \pm 0.3$ ) than a low hs-CRP level TE and non-TE (TE: $0.9 \pm 0.1$, non-TE: $0.6 \pm 0.1$ ). Vegetable consumption of $<91.5 \mathrm{~g} /$ day was highly associated with a high-risk hs-CRP level (adjusted odds ratio (ORs): second tertile (T2): 2.290, (95\% confidence interval (CI): 1.285-4.082); first tertile (T1): 2.474 (95\% CI: 1.368-4.475), $P=0.003$ ) compared with that of the highest (T3) in TE. Low (T1 and T2) vegetable consumption was associated with a 54-63\% increased prevalence of more than two concurrent CVDs risk factors compared with that of the highest in the TE group $(P=0.012)$. Higher vegetable consumption greatly decreased the prevalence of CVDs risk factors by $63-86 \%$ in the low-risk and high-risk hs-CRP TE groups. Our results highlight the cardioprotective effects of higher consumption of vegetables in Korean adults with TE. Evidence-based clinical risk factor management and multifaceted approaches at the community and population levels targeting prevention in high-burden groups are recommended to reduce the premature mortality attributed to CVD.
\end{abstract}

\section{Introduction}

Obesity-induced complications such as hyperlipidemia, hyperglycemia, and hypertension accelerate a lifelong risk of cardiometabolic multimorbidity [1]. The increasing prevalence of cardiometabolic-related deaths makes it the leading cause of death worldwide in individuals under 65 years of age [2].

High-sensitive C-reactive protein (hs-CRP) determines the inflammatory status, and the existence of inflammation is reported to be related to several features of cardiovascular disease (CVD) [3]. High levels of hs-CRP ( $>3 \mathrm{mg} / \mathrm{L})$ cause higher relative cardiovascular risk, which is an underlying pathophysiological process in conditions with a chronic inflammatory status such as obesity, type 2 diabetes (T2DM), and CVD [4]. Independent associations have been reported between hs-CRP concentration and hypertriglyceridemia and the reduced high-density lipoproteincholesterol (HDL-c) level [5], insulin resistance [6], and cardiovascular events [7].

In traditional Korean medicine, the constitution type of individuals can be identified by their physical and psychological traits, including body structure, function, and metabolism [8]. Constitution type has displayed accuracy in recent studies in predicting metabolic syndrome (MetS) [9] and CVD [10]. Particularly, the Tae-eumin (TE) type showed 
a positive association with the components of MetS, including obesity-related factors [11-14] and T2DM [15]. Recent cohort studies have reported lower levels of adiponectin and ghrelin hormone, which act as a regulator of food intake [16], and proatherogenesis [17] in TE. Similarly, the obesity-linked response of food and diet in TE has been reviewed [18].

Lifestyle risk factors including smoking, drinking, inactivity, and unhealthy diet considerably increased the prevalence of onset of MetS and CVDs [19]. Among them, dietary preventive measures act as a modifiable risk factor in the inflammatory pathways [20,21]. A prudent diet high in fruits, vegetables, nuts, and whole grains, which has beneficial effects on the anti-inflammatory capacity by lowering the CRP and CVDs risks, has been recommended in numerous evidence-based studies [22-24].

No study to date has assessed the relationship between dietary factors, hs-CRP concentration, and cardiometabolic risk factors according to constitution types. Therefore, we aimed to elucidate the association of beneficial or harmful dietary factors, serum hs-CRP levels, and cardiometabolic risk factors in Korean adults according to their constitution types.

\section{Materials and Methods}

2.1. Study Design and Population. The Korean Medicine Daejeon Citizen Cohort (KDCC) study [25] is the first prospective ongoing cohort study to assess the associations with lifestyle factors and chronic diseases based on the type of traditional Korean medicine. In the present study, we only analyzed the cross-sectional survey data after obtaining the permission of the Korean Medicine Data Center (KDC) administration.

The participants included individuals aged $\geq 30$ years and $\leq 55$ years who were residents of Daejeon at the time of enrollment in the KDCC between June 2017 and December 2019. Participants were excluded if they (1) had difficulty reading and understanding or following study instructions or (2) were diagnosed with cancer(s) or any CVDs, such as myocardial infarction, angina, or stroke. A total of 2,000 participants were enrolled in the study at baseline. Participants with no clinical outcomes $(n=3)$ and those with less than $500 \mathrm{kcal}$ or more than $5,000 \mathrm{kcal}$ energy intake $(n=14)$ were excluded from the analysis. In total, 1,983 participants were included in the present study. Tae-yang type was not included in the analysis owing to the low rates $(<0.1 \%)$ of all participants. The inflammatory status of the participants was categorized into low-risk $(<3.0 \mathrm{mg} / \mathrm{L})$ or high-risk $(\geq 3.0 \mathrm{mg} /$ $\mathrm{L})$ groups according to their constitution types (Figure 1).

\subsection{Definition of Cardiometabolic Risk Factors and the hs-CRP} Level. Cardiometabolic risk factors were previously categorized by the National Cholesterol Education ProgramAdult Treatment Panel III (NCEP-ATP III) [26], requiring participants to meet more than two of the following clinical criteria: abdominal obesity based on waist circumference (WC) with cutoff points specific to South Koreans (WC $\geq 90 \mathrm{~cm}$ in men and $\geq 85 \mathrm{~cm}$ in women) plus any of the following: elevated triglyceride (TG) levels $\geq 150 \mathrm{mg} / \mathrm{dL}$ or specific treatment for this lipid abnormality; reduced highdensity lipoprotein-cholesterol (HDL-C) level, $<40 \mathrm{mg} / \mathrm{dL}$ in men and $<50 \mathrm{mg} / \mathrm{dL}$ in women or drug treatment for this lipid abnormality; elevated blood pressure (BP), systolic BP $(\mathrm{SBP}) \geq 130$, or diastolic $\mathrm{BP}(\mathrm{DBP}) \geq 85 \mathrm{mmHg}$ or treatment of previously diagnosed hypertension; and the elevated fasting plasma glucose (FPG) level $\geq 100 \mathrm{mg} / \mathrm{dL}$ or previously diagnosed type 2 diabetes.

The participants were categorized into two groups: (1) hs-CRP levels $<3 \mathrm{mg} / \mathrm{L}$ reflecting a low systemic inflammatory status and lower to moderate vascular risk and (2) hs-CRP levels $\geq 3 \mathrm{mg} / \mathrm{L}$ indicating a high systemic inflammatory status and higher vascular risk [4].

2.3. Sasang Constitution Type. The Korean Sasang constitutional diagnostic questionnaire (KS-15) [27] was employed to assess the individual constitution of the participants. The KS-15 questionnaire consists of 15 items of anthropometric awareness of height and weight and six questions of personality and eight symptom-related questions of physiological functions (Cronbach's alpha $=0.630$ ). The KS-15 is a well-validated short-form assessment tool for constitution type that adapts body mass index (BMI) and age- and sex-specific weighted values for higher coincidence with clinical relevance [27]. We defined the two constitution types as TE type or non-TE (So-yangin, SY and So-eumin, $\mathrm{SE})$ type to compare the differences in the dietary factors related to cardiometabolic outcomes and inflammatory status among the three main types of constitution. Previous studies have showed that $20-30 \%$ of Korean adults were categorized into SY or SE types, while almost half of them had TE type. The non-TE type has opposite or different characters in their traits, diet, BMI, disease prevalence, and genetic factors when compared with that of the TE type [28-30].

2.4. Sociodemographic Characteristics, Anthropometric Measurements, and Biochemical Data. Sociodemographic characteristics for the study population, including age, sex, education level, household income, smoking, alcohol consumption, and physical activity, were surveyed at baseline.

Anthropometric measurements to the nearest $0.1 \mathrm{~kg}$ or $0.1 \mathrm{~cm}$ (i.e., height, weight, WC, SBP, and DBP) were taken by trained personnel with the participants dressed lightly without their shoes before height and weight measurement using the measuring station BSM 370 and InBody 770 (Biospace, Korea). BMI was calculated as weight in $\mathrm{kg}$ divided by height in meters squared $\left(\mathrm{m}^{2}\right)$. BP was measured once using an automatic blood pressure cuff (FT-500R PLUS, Jawon Medica, Korea), and it was measured again after 5-10 min of rest, and the mean value of the two measurements was used for analysis. WC was measured with a tape measure (Hoechst mass-Rollfix, Germany) according to World Health Organization guidelines. 


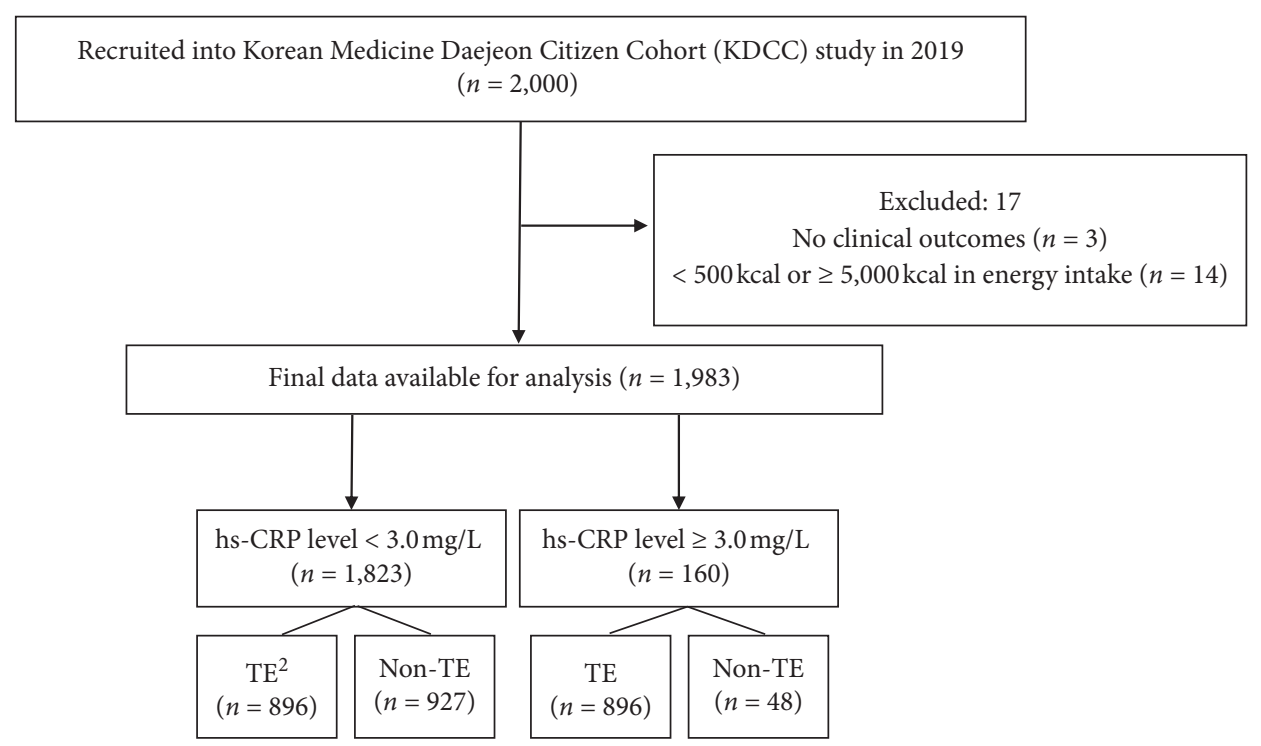

FIGURE 1: Flow diagram illustrating the selection of subjects for analysis. hs-CRP levels $<3 \mathrm{mg} / \mathrm{L}$ reflect a low systemic inflammatory status and lower to moderate vascular risk; whereas, levels $\geq 3 \mathrm{mg} / \mathrm{L}$ indicate high systemic inflammatory status and higher vascular risk. Constitution type was categorized into two groups: TE (Tae-eumin) or non-TE (So-eumin and So-yangin).

Laboratory data (i.e., TG, HDL-C, FPG, and hs-CRP) were recorded after overnight fasting. After $30 \mathrm{~min}$ of incubation, collected blood samples were centrifuged for $10 \mathrm{~min}$ at $3,450 \mathrm{rpm}$, and then, all of the samples were transported to the Seoul Clinical Laboratories (Seoul, Korea) within 24 hours. Serum TG, HDL-C, FPG, and the hs-CRP levels were measured by automated clinical analyzers (ADVIA 1800, Siemens, USA).

\subsection{Short Form of Food Frequency Questionnaires (SF-FFQ).} Dietary intake was assessed using a semiquantitative food frequency questionnaire (FFQ), which was validated by the Korea National Health and Nutrition Examination Survey [25]. Participants were asked to report their frequency (nine options: almost null, 1 time/month, 2-3 times/month, 1-2 times/week, 3-4 times/week, 5-6 times/week, 1 time/day, 2 times/day, or 3 times/day) and portion size (three or four specified portion sizes) of each food item over the past year.

The short form of the FFQ consisted of groups of carbohydrates (rice, mixed grains, noodles, bread, potatoes, and sweet potatoes), proteins (beef, pork, fish, beans, tofu, and eggs), dairy, vegetables, fruits, seaweeds, coffee and tea, alcohol, and fast food. The reported frequency of intake for each food item was converted to daily gram intake of macronutrients as well as total energy using the computeraided nutritional analysis program (CAN Pro, Version 5.0, The Korean Nutrition Society, 2015).

2.6. Statistical Analysis. Descriptive analyses of the participants according to their constitution type and MetS status are presented in Table 1. Group differences were evaluated using chi-square tests for categorical variables including age, sex, education level, household income, smoking status, alcohol consumption, and physical activity level. The results of the one-way analysis of variance and analysis of covariance (ANCOVA) for mean age, BMI, cardiometabolic risk factors, energy intake, nutrients, and food groups are given in Table 1.

We added the data to a multivariate logistic regression model to examine the association between the food frequencies and high-risk hs-CRP level (Table 2) and cardiometabolic risk factors by constitution type (Table 3). Both models ( 1 and 2 ) included several potential confounding factors such as age, sex, BMI, energy intake (kcal), smoking, alcohol consumption, and physical activity [19].

We combined vegetable consumption (lower vs. higher) and the hs-CRP level (high-risk vs. low-risk) to compare the odds ratios (ORs) of the CVD risk factors between the references (vegetable consumption $>91.5 \mathrm{~g} /$ day and hs-CRP $<3.0 \mathrm{mg} / \mathrm{L}$ ) and other combinations of the two clusters (Table 4). Sociodemographic characteristics, including the education level and household income, were fully adjusted to reduce any residual confounding factors in model II.

We performed all analyses using SAS 9.4 (SAS Institute, Cary, North Carolina); all analyses were two-tailed, and a $P$ value of $<0.05$ was considered significant.

\section{Results}

Group differences in general characteristics, CVDs risk factors, and dietary intakes of the subjects according to constitution types with hs-CRP.

A total of 1,983 participants were categorized into lower or higher than $3.0 \mathrm{mg} / \mathrm{L}$ of the hs-CRP level by their constitution types. A total of $11.1 \%$ of TE patients and $4.9 \%$ of non-TE patients had a higher hs-CRP level (TE: $6.6 \pm 0.2$, non-TE: $8.4 \pm 0.3$ ) than the low hs-CRP level TE and non-TE (TE: $0.9 \pm 0.1$, non-TE: $0.6 \pm 0.1$ ).

No significant differences were observed between the four groups in the general characteristics except for sex and smoking $(P<0.05)$. 
TABLE 1: Group differences in general characteristics, CVDs risk factors and dietary intakes of the subjects according to constitution type $\dagger$ with high-sensitive CRP levelł.

\begin{tabular}{|c|c|c|c|c|c|}
\hline \multirow{2}{*}{$\begin{array}{l}\text { hs-CRP level, mg/L } \\
\text { Characteristics }\end{array}$} & \multicolumn{2}{|c|}{ hs-CRP level <3.0 mg/L $(n=1823)$} & \multicolumn{2}{|c|}{ hs-CRP level $\geq 3.0 \mathrm{mg} / \mathrm{L}(n=160)$} & \multirow{2}{*}{$P$ value } \\
\hline & TE $(n=896)$ & Non-TE $(n=927)$ & $\mathrm{TE}(n=112)$ & Non-TE $(n=48)$ & \\
\hline Age (years), (mean \pm SE) & $44.2 \pm 0.2^{\mathrm{a}}$ & $44.8 \pm 0.2^{\mathrm{a}}$ & $42.3 \pm 0.6^{b}$ & $43.7 \pm 1.0^{\mathrm{ab}}$ & 0.001 \\
\hline $30-44$ years $(n, \%)$ & 447 (49.9) & $448(48.3)$ & $71(63.4)$ & $28(58.3)$ & 0.016 \\
\hline $45-55$ years & $274(50.1)$ & $385(51.7)$ & $41(36.6)$ & $20(41.7)$ & \\
\hline \multicolumn{6}{|l|}{ Sex $(\%)$} \\
\hline Male & $339(37.8)$ & $222(23.9)$ & $31(27.7)$ & $13(27.1)$ & $<0.0001$ \\
\hline Female & $557(62.2)$ & $705(76.1)$ & $81(72.3)$ & $35(72.9)$ & \\
\hline Education (\%) & & & & & 0.977 \\
\hline High school lower levels & $318(35.7)$ & $330(35.7)$ & $42(37.8)$ & $17(36.2)$ & \\
\hline College and higher levels & $572(64.3)$ & $594(64.3)$ & $69(62.2)$ & $30(63.8)$ & \\
\hline \multicolumn{5}{|l|}{ Household income $^{1}(\%)$} & 0.977 \\
\hline Low & $170(19.1)$ & $157(17.2)$ & $24(21.4)$ & $7(14.6)$ & \\
\hline Middle & $652(73.2)$ & $662(72.4)$ & $77(68.8)$ & $35(72.9)$ & \\
\hline High & $69(7.7)$ & $96(10.5)$ & $11(9.8)$ & $6(12.5)$ & \\
\hline \multicolumn{5}{|l|}{ Smoking (\%) } & 0.030 \\
\hline No & $777(86.7)$ & $824(88.9)$ & $93(83.0)$ & $47(97.9)$ & \\
\hline Yes & $119(13.3)$ & $103(11.1)$ & $19(17.0)$ & $1(2.1)$ & \\
\hline \multicolumn{5}{|l|}{ Alcohol consumption (\%) } & 0.125 \\
\hline No & $195(35.3)$ & $320(41.4)$ & $93(83.0)$ & $47(97.9)$ & \\
\hline Yes & $358(64.7)$ & $452(58.6)$ & $19(17.0)$ & $1(2.1)$ & \\
\hline \multicolumn{6}{|l|}{ Physical activity $^{2}(\%)$} \\
\hline Low & $275(30.7)$ & $274(29.6)$ & $23(20.5)$ & $18(37.5)$ & 0.067 \\
\hline Moderate & $270(30.1)$ & $297(32.0)$ & $45(40.2)$ & $19(39.6)$ & \\
\hline High & $351(39.2)$ & $356(38.4)$ & $44(39.3)$ & $11(22.9)$ & \\
\hline BMI, $\mathrm{kg} / \mathrm{m}^{2 \S}$ & $26.7 \pm 0.1^{\mathrm{b}}$ & $22.2 \pm 0.1^{\mathrm{c}}$ & $29.3 \pm 0.2^{\mathrm{a}}$ & $22.5 \pm 0.4^{\mathrm{c}}$ & $<0.0001$ \\
\hline \multicolumn{6}{|l|}{ CVDs risk factors $(\text { mean } \pm \mathrm{SE})^{\S}$} \\
\hline $\mathrm{WC}, \mathrm{cm}$ & $88.6 \pm 0.2^{\mathrm{b}}$ & $78.6 \pm 0.2^{\mathrm{c}}$ & $94.5 \pm 0.5^{\mathrm{a}}$ & $79.8 \pm 1.0^{\mathrm{c}}$ & $<0.0001$ \\
\hline Systolic BP, mmHg & $121.3 \pm 0.5^{\mathrm{b}}$ & $115.6 \pm 0.5^{\mathrm{c}}$ & $124.7 \pm 1.4^{\mathrm{a}}$ & $115.7 \pm 2.0^{c}$ & $<0.0001$ \\
\hline Diastolic BP, mmHg & $77.4 \pm 0.5^{\mathrm{a}}$ & $73.0 \pm 0.4^{\mathrm{b}}$ & $78.7 \pm 1.0^{\mathrm{a}}$ & $72.4 \pm 1.6^{\mathrm{b}}$ & $<0.0001$ \\
\hline Fasting plasma glucose, $\mathrm{mmol} / \mathrm{L}$ & $86.6 \pm 0.5^{\mathrm{a}}$ & $83.0 \pm 0.6^{\mathrm{b}}$ & $89.6 \pm 1.5^{\mathrm{a}}$ & $85.6 \pm 2.3^{\mathrm{ab}}$ & $<0.0001$ \\
\hline $\mathrm{HDL}-\mathrm{C}, \mathrm{mmol} / \mathrm{L}$ & $52.2 \pm 0.4^{\mathrm{b}}$ & $58.7 \pm 0.4^{\mathrm{a}}$ & $48.2 \pm 1.2^{\mathrm{c}}$ & $52.8 \pm 1.8^{\mathrm{bc}}$ & $<0.0001$ \\
\hline $\mathrm{TG}, \mathrm{mmol} / \mathrm{L}$ & $156.7 \pm 3.2^{\mathrm{ab}}$ & $130.2 \pm 3.4^{\mathrm{c}}$ & $177.3 \pm 9.1^{\mathrm{a}}$ & $129.9 \pm 13.8^{\mathrm{bc}}$ & $<0.0001$ \\
\hline hs-CRP level, mg/L & $0.9 \pm 0.1^{c}$ & $0.6 \pm 0.1^{\mathrm{d}}$ & $6.6 \pm 0.2^{\mathrm{b}}$ & $8.4 \pm 0.3^{\mathrm{a}}$ & $<0.0001$ \\
\hline$\geq 2$ concurrent CVDs risk factors & $442(49.3)$ & $143(15.4)$ & $77(68.8)$ & $10(20.8)$ & $<0.0001$ \\
\hline \multicolumn{6}{|l|}{ Energy $(\mathrm{kcal} / \mathrm{d})^{3}$} \\
\hline Men & $2238.5 \pm 35.9$ & $2283.6 \pm 44.5$ & $2244.5 \pm 118.8$ & $2409.4 \pm 183.7$ & 0.724 \\
\hline Women & $2082.2 \pm 29.4$ & $2038.8 \pm 26.2$ & $2117.0 \pm 77.4$ & $2253.8 \pm 117.4$ & 0.211 \\
\hline \multicolumn{6}{|l|}{ Nutrients } \\
\hline Carbohydrates (g) & $314.9 \pm 7.3$ & $321.0 \pm 4.9$ & $313.1 \pm 5.3$ & $339.9 \pm 8.1$ & 0.828 \\
\hline Fat $(g)$ & $53.9 \pm 1.7$ & $53.9 \pm 1.1$ & $51.6 \pm 1.0$ & $53.8 \pm 1.7$ & 0.418 \\
\hline Protein (g) & $72.3 \pm 1.9$ & $72.5 \pm 1.2$ & $69.6 \pm 1.2$ & $73.6 \pm 1.9$ & 0.636 \\
\hline $\mathrm{C}: \mathrm{F}: \mathrm{P}(\%)$ & $59.8: 22.7: 13.6$ & $59.8: 22.5: 13.5$ & $60.6: 22.5: 13.4$ & $60.9: 21.9: 13.4$ & $\mathrm{~N} / \mathrm{S}$ \\
\hline
\end{tabular}


TABLE 1: Continued.

\begin{tabular}{|c|c|c|c|c|c|}
\hline \multirow{2}{*}{$\begin{array}{l}\text { hs-CRP level, mg/L } \\
\text { Characteristics }\end{array}$} & \multicolumn{2}{|c|}{ hs-CRP level $<3.0 \mathrm{mg} / \mathrm{L}(n=1823)$} & \multicolumn{2}{|c|}{ hs-CRP level $\geq 3.0 \mathrm{mg} / \mathrm{L}(n=160)$} & \multirow{2}{*}{$P$ value } \\
\hline & TE $(n=896)$ & Non-TE $(n=927)$ & $\mathrm{TE}(n=112)$ & Non-TE $(n=48)$ & \\
\hline \multicolumn{6}{|l|}{ Food groups $(\mathrm{g} / \mathrm{d})^{4 \S \S}$} \\
\hline White rice & $191.7 \pm 2.6$ & $193.4 \pm 2.7$ & $183.7 \pm 7.3$ & $205.4 \pm 11.1$ & 0.385 \\
\hline Wholegrains & $78.5 \pm 2.7$ & $74.9 \pm 2.8$ & $88.7 \pm 7.6$ & $84.5 \pm 11.0$ & 0.290 \\
\hline Noodles and bread & $102.4 \pm 3.1$ & $108.3 \pm 3.2$ & $96.8 \pm 8.8$ & $91.3 \pm 13.3$ & 0.284 \\
\hline Potatoes and sweet potatoes & $46.4 \pm 2.4$ & $45.9 \pm 2.5$ & $37.5 \pm 6.9$ & $48.4 \pm 10.5$ & 0.662 \\
\hline Beans and tofu & $28.5 \pm 1.0$ & $26.9 \pm 1.0$ & $28.0 \pm 2.8$ & $25.0 \pm 4.2$ & 0.622 \\
\hline Fish & $6.5 \pm 0.3$ & $6.3 \pm 0.3$ & $5.0 \pm 0.8$ & $6.5 \pm 1.1$ & 0.285 \\
\hline Beef pork & $60.1 \pm 1.9$ & $57.4 \pm 2.0$ & $56.1 \pm 5.3$ & $58.8 \pm 8.1$ & 0.739 \\
\hline Poultry & $34.7 \pm 1.4$ & $31.9 \pm 1.5$ & $28.7 \pm 4.0$ & $31.1 \pm 6.1$ & 0.347 \\
\hline Eggs & $36.7 \pm 1.2$ & $34.9 \pm 1.3$ & $33.8 \pm 3.4$ & $29.7 \pm 5.2$ & 0.445 \\
\hline Vegetables and mushrooms & $54.3 \pm 1.1^{\mathrm{a}}$ & $52.5 \pm 1.1^{\mathrm{ab}}$ & $45.1 \pm 3.1^{\mathrm{b}}$ & $57.6 \pm 4.7^{\mathrm{ab}}$ & 0.025 \\
\hline Fruits & $84.7 \pm 3.4$ & $91.0 \pm 3.5$ & $73.5 \pm 9.5$ & $77.3 \pm 14.2$ & 0.214 \\
\hline Milk and yogurt & $101.4 \pm 3.8$ & $102.3 \pm 4.0$ & $119.3 \pm 10.9$ & $100.3 \pm 16.4$ & 0.481 \\
\hline Coffee and Tea & $125.2 \pm 3.6$ & $123.4 \pm 3.7$ & $136.5 \pm 9.9$ & $122.4 \pm 15.4$ & 0.659 \\
\hline Beer and soju & $45.3 \pm 2.6$ & $53.4 \pm 2.7$ & $47.6 \pm 7.3$ & $39.2 \pm 10.7$ & 0.111 \\
\hline Hamburger and pizza & $88.4 \pm 4.5$ & $88.4 \pm 4.5$ & $95.8 \pm 12.6$ & $94.1 \pm 19.7$ & 0.771 \\
\hline
\end{tabular}

${ }^{\dagger}$ Constitution type was categorized into two groups as TE (Tae-eumin) and non-TE (So-eumin and So-yangin). ${ }^{*}$ High-sensitive CRP levels were categorized into two different groups: hs-CRP level $<3.0 \mathrm{mg} / \mathrm{L}$ and hs-CRP level $\geq 3.0 \mathrm{mg} / \mathrm{L} .{ }^{1}$ Monthly household income was divided into 3 groups: low ( $>3,000,000$ won), middle (3,000,000-7,000,000 won), and high (7,000,000 won $\leq) .{ }^{2}$ Physical activity: total MET minutes per week: low ( $<600 \mathrm{MET}$-min/wk); moderate (600-1,500 MET-min/wk); and high (1,500 MET-min/wk). $P$ values were obtained from Rao-Scott chi-square tests for categorical variables and Bonferroni

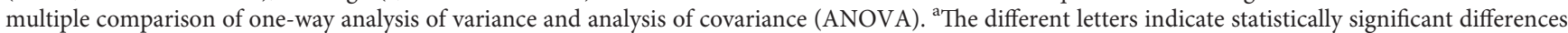
$(P<0.05)$, analyzed using ANCOVA followed by Bonferroni's multiple comparision test. ${ }^{\varsigma}$ Least square means-sq adjusted for age and sex. CVDs, cardiovascular diseases; hs-CRP, high-sensitive C-reactive protein; BMI, body mass index; WC, waist circumference; BP, blood pulse; HDL-C, high-density lipoprotein cholesterol; TG, triglycerides. ${ }^{3}$ Adjusted age only. ${ }^{\circledR}$ Least square means-sq adjusted for age, sex, and energy intake (kcal). ${ }^{4}$ Food groups were surveyed using short-form of the food frequency questionnaires (FFQ) which includes grains (white rice, wholegrains, noodles bread, potatoes, and sweet potatoes) proteins (bean, tofu, fish, beef, pork, poultry, and eggs); vegetables (cabbage, radish, carrot, zucchini, and mushrooms); fruits (apples, banana, persimon, strawberries, and pear); dairy (milk and yogurt); drink (coffee and tea); alcohol (beer and soju); fast foods (hamburger and pizza).

TABle 2: Associations between fruits, vegetables, fish and meats consumptions and high-risk hs-CRP level of the subjects by constitution types.

\begin{tabular}{|c|c|c|c|c|c|}
\hline \multirow{2}{*}{ Food groups } & \multirow{2}{*}{$\begin{array}{l}\text { hs-CRP level } \\
\geq 3.0 \mathrm{mg} / \mathrm{L}\end{array}$} & \multicolumn{2}{|c|}{ Model 1 OR (95\% CI) } & \multicolumn{2}{|c|}{ Model 2 OR $(95 \%$ CI $)$} \\
\hline & & TE & Non-TE & $\mathrm{TE}$ & Non-TE \\
\hline \multirow{4}{*}{$\begin{array}{l}\text { Fruits } \\
\text { Adjusted OR (95\% CI) }\end{array}$} & & & & & \\
\hline & & $(\mathrm{Ref}=\mathrm{T} 3: 155.5 \mathrm{~g} /$ day $)$ & & $(\operatorname{Ref}=\mathrm{T} 3: 155.5 \mathrm{~g} /$ day $)$ & \\
\hline & T2: $59.6 \mathrm{~g} /$ day & $1.116(0.658-1.893)$ & $0.911(0.458-1.811)$ & $1.158(0.673-1.994)$ & $0.976(0.472-2.018)$ \\
\hline & $\mathrm{T} 1: 17.8 \mathrm{~g} /$ day & $0.940(0.543-1.627)$ & $0.712(0.327-1.549)$ & $0.976(0.552-1.727)$ & $0.899(0.400-2.019)$ \\
\hline$P$ value for trend & & 0.923 & 0.441 & 0.954 & 0.822 \\
\hline \multicolumn{6}{|l|}{ Vegetables } \\
\hline Adjusted OR (95\% CI) & & $(\mathrm{Ref}=\mathrm{T} 3: 91.5 \mathrm{~g} /$ day $)$ & & $(\mathrm{Ref}=\mathrm{T} 3: 91.5 \mathrm{~g} /$ day $)$ & \\
\hline & $\mathrm{T} 2: 50.3 \mathrm{~g} /$ day & $2.169(1.235-3.809)$ & $0.651(0.320-1.322)$ & $2.290(1.285-4.082)$ & $0.645(0.308-1.350)$ \\
\hline & $\mathrm{T} 1: 21.2 \mathrm{~g} / \mathrm{day}$ & $2.488(1.401-4.421)$ & $0.634(0.300-$ & $2.474(1.368-4.475)$ & $0.564(0.257-$ \\
\hline$P$ value for trend & & 0.002 & 0.210 & 0.003 & 0.140 \\
\hline \multirow{4}{*}{$\begin{array}{l}\text { Fish } \\
\text { Adjusted OR (95\% CI) }\end{array}$} & & & & & \\
\hline & & $(\mathrm{Ref}=\mathrm{T} 3: 11.4 \mathrm{~g} /$ day $)$ & & $(\mathrm{Ref}=\mathrm{T} 3: 11.4 \mathrm{~g} / \mathrm{day})$ & \\
\hline & T2: $4.0 \mathrm{~g} /$ day & $1.578(0.914-2.724)$ & $1.069(0.518-2.205)$ & $1.664(0.948-2.921)$ & $1.043(0.489-2.226)$ \\
\hline & $\mathrm{T} 1: 1.9 \mathrm{~g} /$ day & $1.564(0.900-2.718)$ & $0.931(0.445-$ & $1.562(0.882-2.769)$ & $0.948(0.436-2.063)$ \\
\hline $\begin{array}{l}P \text { value for trend } \\
\text { Beef and pork }\end{array}$ & & 0.080 & 0.931 & 0.080 & 0.948 \\
\hline \multirow[t]{3}{*}{ Adjusted OR (95\% CI) } & & $(\mathrm{Ref}=\mathrm{T} 3: 87.0 \mathrm{~g} /$ day $)$ & & $(\mathrm{Ref}=\mathrm{T} 3: 87.0 \mathrm{~g} /$ day $)$ & \\
\hline & $\mathrm{T} 2: 39.1 \mathrm{~g} /$ day & $1.190(0.695-2.039)$ & $0.777(0.367-1.644)$ & $1.090(0.627-1.894)$ & $0.798(0.363-1.752)$ \\
\hline & $\mathrm{T} 1: 17.4 \mathrm{~g} / \mathrm{day}$ & $0.922(0.523-1.626)$ & $0.645(0.290-1.435)$ & $0.836(0.465-1.504)$ & $0.634(0.269-1.493)$ \\
\hline$P$ value for trend & & 0.934 & 0.277 & 0.668 & 0.305 \\
\hline
\end{tabular}

Model I, age, sex, BMI, and energy intake (kcal). Model II: model I + smoking, alcohol consumption, and physical activity. Boldface type indicates statistical significance. 
TABle 3: Associations of cardiometabolic risk factors and tertiles of the vegetables consumption of the subjects by constitution types.

\begin{tabular}{|c|c|c|c|c|c|}
\hline \multirow{3}{*}{ CVDs risk factors } & \multicolumn{5}{|c|}{ Vegetables consumption } \\
\hline & & \multicolumn{2}{|c|}{ Model 1 OR (95\% CI) } & \multicolumn{2}{|c|}{ Model 2 OR (95\% CI) } \\
\hline & & $\mathrm{TE}$ & Non-TE & $\mathrm{TE}$ & Non-TE \\
\hline \multirow[t]{3}{*}{ High $\mathrm{BP}(\mathrm{SBP} \geq 130$ or $\mathrm{DBP} \geq 85 \mathrm{mmHg})$} & & \multicolumn{4}{|c|}{$(\mathrm{Ref}=\mathrm{T} 3: 91.5 \mathrm{~g} / \mathrm{day})$} \\
\hline & $\mathrm{T} 2$ & $1.397(0.984-1.983)$ & $0.868(0.554-1.360)$ & $1.370(0.956-1.963)$ & $0.862(0.544-1.365)$ \\
\hline & $\mathrm{T} 1$ & $1.254(0.874-1.801)$ & $1.412(0.909-2.193)$ & $1.258(0.868-1.823)$ & $1.389(0.880-2.180)$ \\
\hline$P$ value for trend & & 0.211 & 0.119 & 0.219 & 0.150 \\
\hline \multirow[t]{3}{*}{ Elevated FPG ( $\geq 100 \mathrm{mg} / \mathrm{dL})$} & \multicolumn{5}{|c|}{$(\mathrm{Ref}=\mathrm{T} 3: 91.5 \mathrm{~g} / \mathrm{day})$} \\
\hline & $\mathrm{T} 2$ & $1.465(0.881-2.436)$ & $0.804(0.326-1.988)$ & $1.414(0.837-2.389)$ & $0.803(0.322-2.002)$ \\
\hline & $\mathrm{T} 1$ & $0.853(0.483-1.506)$ & $1.416(0.608-3.301)$ & $0.789(0.435-1.431)$ & $1.405(0.595-3.319)$ \\
\hline$P$ value for trend & & 0.655 & 0.424 & 0.506 & 0.436 \\
\hline \multirow[t]{3}{*}{ Elevated TG ( $\geq 150 \mathrm{mg} / \mathrm{dL})$} & \multicolumn{5}{|c|}{$(\mathrm{Ref}=\mathrm{T} 3: 91.5 \mathrm{~g} / \mathrm{day})$} \\
\hline & $\mathrm{T} 2$ & $1.146(0.816-1.610)$ & $0.809(0.512-1.279)$ & $1.165(0.819-1.657)$ & $0.856(0.535-1.369)$ \\
\hline & $\mathrm{T} 1$ & $1.051(0.740-1.493)$ & $1.273(0.805-2.014)$ & $1.130(0.786-1.623)$ & $1.317(0.818-2.119)$ \\
\hline$P$ value for trend & & 0.768 & 0.294 & 0.502 & 0.252 \\
\hline \multirow[t]{3}{*}{$\begin{array}{l}\text { Reduced HDL-C }(<40 \mathrm{mg} / \mathrm{dL} \text { in men and } \\
<50 \mathrm{mg} / \mathrm{dL} \text { in women })\end{array}$} & \multicolumn{5}{|c|}{$(\operatorname{Ref}=\mathrm{T} 3: 91.5 \mathrm{~g} / \mathrm{day})$} \\
\hline & $\mathrm{T} 2$ & $1.017(0.715-1.448)$ & $0.929(0.604-1.431)$ & $1.146(0.795-1.652)$ & $1.0732(0.684-1.683)$ \\
\hline & $\mathrm{T} 1$ & $1.118(0.779-1.602)$ & $0.745(0.466-1.191)$ & $1.238(0.852-1.799)$ & $0.841(0.515-1.372)$ \\
\hline \multirow{4}{*}{$\begin{array}{l}\text { High WC ( } \geq 90 \mathrm{~cm} \text { in men and } \geq 85 \mathrm{~cm} \text { in } \\
\text { women) }\end{array}$} & & 0.549 & 0.223 & 0.261 & 0.503 \\
\hline & \multicolumn{5}{|c|}{$(\mathrm{ref}=\mathrm{T} 3: 91.5 \mathrm{~g} / \mathrm{day})$} \\
\hline & $\mathrm{T} 2$ & $1.186(0.797-1.765)$ & $1.059(0.481-2.333)$ & $1.254(0.832-1.890)$ & $0.864(0.379-1.967)$ \\
\hline & $\mathrm{T} 1$ & $1.166(0.774-1.758)$ & $1.309(0.600-2.855)$ & $1.259(0.824-1.924)$ & $0.989(0.434-2.254)$ \\
\hline$P$ for trend & & 0.453 & 0.490 & 0.278 & 0.997 \\
\hline \multirow[t]{3}{*}{$\geq 2$ concurrent CVDs risk factors } & \multicolumn{5}{|c|}{$(\operatorname{Ref}=\mathrm{T} 3: 91.5 \mathrm{~g} /$ day $)$} \\
\hline & $\mathrm{T} 2$ & $1.468(1.023-2.109)$ & $0.906(0.564-1.455)$ & $1.542(1.061-2.243)$ & $0.953(0.587-1.550)$ \\
\hline & $\mathrm{T} 1$ & $1.541(1.058-2.244)$ & $1.099(0.677-1.787)$ & $1.631(1.106-2.404)$ & $1.114(0.673-1.844)$ \\
\hline$P$ value for trend & & 0.023 & 0.709 & 0.012 & 0.679 \\
\hline
\end{tabular}

Model I, age, sex, BMI, and energy intake (kcal). Model II, model I + smoking, alcohol consumption, and physical activity. Boldface type indicates statistical significance.

TABLE 4: Multivariate logistic regression between vegetables consumption, hs-CRP level and cardiometabolic risk factors by constitution type.

\begin{tabular}{|c|c|c|c|c|}
\hline & \multirow{2}{*}{ Vegetables consumption } & \multirow{2}{*}{ hs-CRP level } & \multicolumn{2}{|c|}{$\geq 2$ concurrent CVDs risk factors } \\
\hline & & & TE & Non-TE \\
\hline \multirow[t]{4}{*}{ Model I OR (95\% CI) } & (Ref, <91.5 g/day) & $(\mathrm{Ref}, \geq 3.0 \mathrm{mg} / \mathrm{L})$ & \multicolumn{2}{|r|}{-} \\
\hline & Lower & Low-risk & $0.553(0.305-1.006)$ & $0.501(0.178-1.406)$ \\
\hline & Higher & High-risk & $0.248(0.064-0.961)$ & $0.724(0.123-4.275)$ \\
\hline & Higher & Low-risk & $0.368(0.197-0.691)$ & $0.434(0.141-1.339)$ \\
\hline \multirow[t]{4}{*}{ Model II OR (95\% CI) } & (Ref, <91.5 g/day) & $(\mathrm{Ref}, \geq 3.0 \mathrm{mg} / \mathrm{L})$ & \multicolumn{2}{|l|}{ ( } \\
\hline & Lower & Low-risk & $0.557(0.305-1.018)$ & $0.625(0.206-1.895)$ \\
\hline & Higher & High-risk & $0.141(0.028-0.695)$ & $0.695(0.054-8.948)$ \\
\hline & Higher & Low-risk & $0.361(0.190-0.684)$ & $0.548(0.161-1.859)$ \\
\hline
\end{tabular}

Model I, age, sex, BMI, energy intake (kcal), smoking, alcohol consumption, and physical activity. Model II, model I + education level and household income. Boldface type indicates statistical significance.

The risk factors for CVD, BMI $\left(\mathrm{kg} / \mathrm{m}^{2}\right)$, WC $(\mathrm{cm})$, SBP $(\mathrm{mmHg}), \mathrm{DBP}(\mathrm{mmHg}), \mathrm{FPG}(\mathrm{mg} / \mathrm{dL})$, and TG $(\mathrm{mg} / \mathrm{dL})$ were the highest $(P<0.0001)$, while HDL-C $(\mathrm{mg} / \mathrm{dL})$ was the lowest in the high TE group $(P<0.0001)$ (Table 1$)$.

No significant group differences were found in the energy intake ( $\mathrm{kcal}$ ) or nutrient intake between the four groups. Regarding the food groups $(\mathrm{g} / \mathrm{d})$, in vegetables, mushrooms were highest in the low hs-CRP TE group and lowest in the high hs-CRP TE group (low TE: $54.3 \pm 1.1$ vs. high TE: $45.1 \pm 3.1, P=0.025)$ after adjusting for age, sex, and energy intake (kcal).
3.1. Associations between Fruits, Vegetables, and Red Meats Consumption and the hs-CRP Level of the Subjects by Constitution Types. A covariate-adjusted multiple logistic regression analysis was performed to assess the beneficial or harmful association between tertiles of fruits, vegetables, and red meats and the hs-CRP level. No significant association between tertiles of fruits or meats and the hs-CRP level was observed in the TE and non-TE groups after adjusting for age, sex, BMI, energy intake (kcal) (model 1), and model 2 (model 1+ smoking, alcohol consumption, and physical activity) (Table 2). 
In the tertile of vegetables consumption, an approximately 2.5-fold higher risk of hs-CRP (Hs-CRP level $\geq 3.0 \mathrm{mg} / \mathrm{L}$ ) was seen in tertiles 1 (T1: $21.2 \mathrm{~g} /$ day) and 2 (T2: $50.3 \mathrm{~g} /$ day) groups compared with the highest group (T3: 91.5 g/day) in TE (adjusted ORs: T2: 2.290 (95\% confidence interval (CI): 1.285-4.082); T1: 2.474 (95\% CI: 1.368-4.475), $P=0.003)$.

\subsection{Associations of Cardiometabolic Risk Factors and Tertiles} of Vegetable Consumption of the Subjects by Constitutional Types. The associations between tertiles of vegetable consumption and cardiometabolic risk factors are presented in Table 3. No statistical significance was observed in the cardiometabolic risk factors such as elevated BP, FPG, TG, reduced $\mathrm{HDL}-\mathrm{c}$, and high $\mathrm{WC}$; in contrast, individuals with more than two concurrent those of CVDs risk factors showed significant associations. The multivariate-adjusted ORs of the prevalence of more than two concurrent CVDs risk factors and the lowest (T1) vegetable consumption was 1.631 (95\% CI: 1.106-2.404, $P=0.012$ ) in TE (Table 3).

3.3. Multivariate Logistic Regression between Vegetable Consumption, hs-CRP Level, and Cardiometabolic Risk Factors by Constitution Types. The multivariate-adjusted regression models (models I and II) showed that higher vegetable consumption greatly decreases the prevalence of CVDs risk factors in model I (OR: 0.248, 95\% CI: 0.064-0.961) and model II (OR: 0.141, 95\% CI: 0.028-0.695) in the high-risk hs-CRP TE group. Similarly, significant ORs of the prevalence of CVDs risk factors were estimated in the low-risk hs-CRP TE group (Table 4).

\section{Discussion}

This study examined the association between dietary factors, hs-CRP level, and cardiometabolic risk factors in Korean adults according to their constitution types. Overall, $8.1 \%$ of the participants in the present study had high-risk hs-CRP levels. Our results illustrate that lower consumption of vegetables $(<92 \mathrm{~g} / \mathrm{d})$ is highly associated with the prevalence of having a high-risk hs-CRP level (adjusted ORs: T2: 2.290 (95\% CI: 1.285-4.082), T1: 2.474 (95\% CI: 1.368-4.475), $P=0.003)$ in the TE group. Furthermore, the lowest (T1) of vegetable consumption had a $63 \%$ increased prevalence of more than two concurrent CVD risk factors compared with that of the highest in the TE group $(P=0.012)$. Last, higher vegetable consumption greatly decreased the prevalence of CVD risk factors by $63-86 \%$ in the low-risk and high-risk hs-CRP TE groups.

A previous meta-analysis reported a positive association between active smoking and MetS (RR: 1.26, 95\% CI) [31]. Another cross-sectional study found an increased risk of high TGs, low HDL-C, and a decreased risk of high BP compared with nonsmokers [32]. However, this could not elucidate the interrelationship directly owing to disparities in the extent of the contribution of sex differences and inaccurate self-reported smoking status without cotinineverified smoking status in this study. Therefore, we used smoking status with other lifestyle-related (alcohol consumption, physical activity, and energy intake) variables including age and sex as potential confounding factors for the present study.

An elevated hs-CRP level, which is the most standardized inflammatory marker, predicts the risk for developing MetS and CVDs. The association between MetS and its cluster with hs-CRP is consistent with the findings of previous studies. A Korean population-based study found that the hs-CRP level plays a major role in the development of MetS [5]. In women, obesity status affects the inflammatory status, while BMI was considered the most significant risk factor in men. Regardless of the positive association between the hs-CRP level and hyper TG, low HDL-C levels were observed. Similarly, these traditional markers including WC and lipid parameters such as TG, HDL-C, and LDL-C levels were significantly related to the hs-CRP level not only in obese females [33, 34] but also that of adolescent girls [35]. In the Bogalusa heart study [36], the elevated hs-CRP level was associated with follow-up HOMA-IR and increased risk of T2DM in nondiabetic adults. This finding suggests that there is a combined effect of inflammation and insulin resistance on the pathogenesis of hyperglycemia. In fact, a previous U.S. population study found that the elevated hsCRP group had more CVD with MetS [37]. It has also been predicted that there is a 1.48-times higher MetS risk in the hs-CRP increased group than in that of their counterparts in healthy subjects [38].

Consistently, $39.7 \%$ of the participants had high-risk hsCRP, and the highest prevalence of the predictors of CVD was found in the TE type [39]. A previous study found that TE type itself was a "risk factor" for an increasingly higher odds ratio (adjusted ORs: 1.99, 95\% CI: 1.60-2.47) of the high-risk hs-CRP compared with non-TE types (SY or SE).

Our results support the impression of differences between the TE and non-TE groups; the TE group had a higher BMI as well as more CVDs risk factors such as WC, BP, FPG, and TG and reduced HDL-c compared with those in the non-TE group. The development of MetS in the TE group is likely due to dysregulated metabolic homeostasis underlying CVD-related pathogenesis, such as dyslipidemia or elevated BP. Hence, it is important to identify individuals who have a high risk of CVD by reducing MetS and CVD-related mortality.

Our results highlight the harmful effects of low vegetable consumption on the high-risk hs-CRP level groups as well as the cardiometabolic risk factors in the TE group. This is in agreement with a previous study that reported the association of low adherence to the traditional Mediterranean diet with inflammatory biomarkers in the adult population [40, 41]. Similarly, a large population-based study reported an inverse association between high intake of a vegetableseafood dietary pattern and components of MetS and CRP but found a positive association between high intake of a meat-instant food dietary pattern and components of MetS and CRP [22]. Another population-based prospective study observed that a proinflammatory diet represents a potential MetS risk factor in 157,812 Korean adults (mean age 52.8 years; mean follow-up of 7.4 years) [42]. Additionally, a 
systematic review found that CRP and meat-based or Western-like dietary patterns were positively associated while vegetable- and fruit-based or healthy patterns were negatively associated after adjusting for potential confounders [23].

A choice of healthy foods such as fruits, vegetables, whole grains, nuts, seeds, and legumes has beneficial effects in lowering inflammation, whereas a Western dietary pattern that includes red meat with saturated fatty acids is associated with excessive production of proinflammatory cytokines in the body [20]. In the present study, no group differences were found in energy intake, macronutrients, and food groups except vegetables between the participants. This reflects, even in the metabolically high-risk type (normally, TE), that no specific problematic eating related to inflammation was observed in their diet. Therefore, future studies are needed to assess the dietary risk factors for secondary prevention in the development of MetS or CVD.

Importantly, obesity-induced metabolic inflammation produces CRP, as a proinflammatory biomarker, and adipokines, including tumor necrosis factor-alpha (TNF- $\alpha$ ) and interleukin (IL)-6, which further propagate inflammation [43]. An inappropriate diet, such as high sugar-sweetened beverages and low fruit and vegetable intake, is related to the development of a proinflammatory state, which leads to cardiometabolic diseases $[44,45]$. In contrast, an antiinflammatory nutrient-based diet has complex dietary bioactive properties, such as fiber, polyphenols, and vitamins that promote anti-inflammatory and cardioprotective effects $[46,47]$. In fact, a higher fiber intake $(\geq 15 \mathrm{~g} / 1,000 \mathrm{kcal})$ was associated with lower $\mathrm{HbAlc}$ and CRP than lower fiber intake in 1,785 middle-aged people with type 2 diabetes [48]. Furthermore, a significant inverse association was observed between total vegetable consumption and TNF- $\alpha$ level $(-0.078 ; 95 \% \mathrm{CI},-0.151$ to -0.005$)$ in obese older adults [24]. Another previous study found that $7.1 \%$ weight loss, $22.3 \%$ visceral adiposity reduction, $15.5 \%$ improvement in HOMAIR and lipid profiles, and inflammatory biomarkers (hs-CRP, $-29.5 \%$; IL-6, $-18.2 \%$; TNF- $\alpha,-34.2 \%)$ following energyrestricted anti-inflammatory diet therapy for patients with nonalcoholic fatty liver disease [49].

In line with this, we found a significant protective effect of high vegetable consumption on cardiometabolic risk factors in the high-risk and low-risk hs-CRP level TE groups. Several large population-based studies have reported that there is an inherent metabolic high-risk associated with the components of MetS in TE [9-14]. In particular, high BMI and obesity-related metabolic risks, such as central obesity (WC and WHR) $[9,11,50]$, which are interrelated to the development of insulin resistance [14] and T2DM [15], were found in the TE group. Obesity is known to produce a chronic low-grade inflammatory status, which is the pathogenesis of MetS and the progression of CVD. For this reason, early detection and specific treatment of CVDs are essential for patients with obesity.

The main strengths of our study are that it uses data from a nationally representative survey of Korean adults and that it is the first study to investigate associations between dietary risk factors related to the inflammatory status and CVD risk factors by constitution types. Nevertheless, we detected only $<10 \%$ of the high-risk level of hs-CRP in the present study samples (between unequal sample size); therefore, one needs to be careful when interpreting the data. This study also had several limitations. First, the design of this study was crosssectional, permitting to make projections to the odds of the prevalence of CVD. Second, we used only serum hs-CRP levels to assess the inflammatory status. Third, we employed a short form of the FFQ to assess dietary factors for the participants' convenience. Except for vegetable consumption, we could not find any other beneficial or harmful dietary factors related to the hs-CRP level or CVD risk factors in Korean adults. Further research is warranted to evaluate overall diet or dietary patterns to explore dietrelated cardiometabolic risk factors in the inherent metabolically high-risk Korean population.

\section{Conclusions}

In summary, this study demonstrates the existence of a significant relationship between the dietary factors, hs-CRP level, and cardiometabolic risk factors in Korean adults based on the constitution types. The present data suggest that higher consumption of vegetables provides cardioprotective effects in Korean adults with TE. Evidencebased clinical risk factor management and multifaceted approaches at the community and population levels targeting prevention in high-burden groups are strongly needed to reduce premature mortality from CVD.

\section{Data Availability}

The data that support the findings of this study are available from the web-based KDC electronic data capture system by the Korean Medicine Data Center (KDC) of the KIOM, but restrictions apply to the availability of these data, which were used under license for the current study, and so are not publicly available.

\section{Ethical Approval}

The Institutional Review Board at the Korea Institute of Oriental Medicine (KIOM) and Dunsan Korean Medicine Hospital of Daejeon University reviewed and approved this study (IRB No. I-1703/002-002, DJDSKH-17-BM-12).

\section{Consent}

Informed consent was obtained from all participants when data were collected.

\section{Disclosure}

The funding bodies have no role in designing the study, collection, analysis, and interpretation of data and in writing the article.

\section{Conflicts of Interest}

The authors declare that they have no conflicts of interest. 


\section{Authors' Contributions}

This project was conceived by S.L and Y.B. S.L. contributed to the funding acquisition. J.K. made the conceptualization and design of the work. K.J. curated and managed the data. J.K. performed the statistical analysis, interpreted data, and drafted the article. Y.B. carried out critical revision and improved the manuscript. Y.B. and S.L. had given the final approval of the article. All authors read and approved the final draft of the article.

\section{Acknowledgments}

The authors thank all the contributions of the study investigators and participants for their great work. This study was supported by the "Development of Korean Medicine Original Technology for Preventive Treatment Based on Integrative Big Data" grant from the Korea Institute of Oriental Medicine (KSN2022120).

\section{References}

[1] M. Kivimäki, E. Kuosma, J. E. Ferrie et al., "Overweight, obesity, and risk of cardiometabolic multimorbidity: pooled analysis of individual-level data for 120813 adults from 16 cohort studies from the USA and Europe," The Lancet Public Health, vol. 2, no. 6, pp. e277-e285, 2017.

[2] N. S. Shah, "Adverse trends in premature cardiometabolic mortality in the United States, 1999 to 2018," Journal of the American Heart Association, vol. 9, no. 23, e018213 pages, 2020.

[3] P. M. Ridker, "A test in context," Journal of the American College of Cardiology, vol. 67, no. 6, pp. 712-723, 2016.

[4] C. Vlachopoulos, P. Xaplanteris, V. Aboyans et al., "The role of vascular biomarkers for primary and secondary prevention. A position paper from the European society of cardiology working group on peripheral circulation," Atherosclerosis, vol. 241, no. 2, pp. 507-532, 2015.

[5] Y. Song, S. K. Yang, J. Kim, and D.-C. Lee, "Association between C-reactive protein and metabolic syndrome in Korean adults," Korean Journal of Family Medicine, vol. 40, no. 2, pp. 116-123, 2019.

[6] V. Wieser, A. R. Moschen, and H. Tilg, "Inflammation, cytokines and insulin resistance: a clinical perspective," Archivum immunologiae et therapiae experimentalis, vol. 61, no. 2, pp. 119-125, 2013.

[7] G. K. Hansson, P. Libby, and I. Tabas, "Inflammation and plaque vulnerability," Journal of Internal Medicine, vol. 278, no. 5, pp. 483-493, 2015.

[8] K. H. Song, S.-G. Yu, and J. Y. Kim, "Prevalence of metabolic syndrome according to sasang constitutional medicine in Korean subjects," Evidence-Based Complementary and Alternative Medicine, vol. 2012, Article ID 646794, 8 pages, 2012.

[9] S. Lee, "Sasang constitutional types for the risk prediction of metabolic syndrome: a 14-year longitudinal prospective cohort study," BMC Complementary and Alternative Medicine, vol. 17, no. 1, p. 438, 2017.

[10] N. H. Cho, J. Y. Kim, S. S. Kim, and C. Shin, "The relationship of metabolic syndrome and constitutional medicine for the prediction of cardiovascular disease," Diabetes \& Metabolic Syndrome: Clinical Research \& Reviews, vol. 7, no. 4, pp. 226-232, 2013.
[11] E. Jang, "Could the Sasang constitution itself be a risk factor of abdominal obesity?" BMC Complementary and Alternative Medicine, vol. 13, no. 1, p. 72, 2013.

[12] J.-E. Park, C. Shin, and S. Lee, "Effect of lifestyle factors on hypertension by constitution type: a large community-based study," Evidence-Based Complementary and Alternative Medicine, vol. 2019, Article ID 3231628, 8 pages, 2019.

[13] J. Lee, H. Jang, B. Park, E. Lee, B. Koh, and J. Lee, "Can the sasang constitutional type trait act as an independent risk factor for dyslipidemia?" Journal of Korean Medicine for Obesity Research, vol. 14, no. 2, pp. 63-71, 2014.

[14] K. Choi, J. Lee, J. Yoo, E. Lee, B. Koh, and J. Lee, "Sasang constitutional types can act as a risk factor for insulin resistance," Diabetes Research and Clinical Practice, vol. 91, no. 3, pp. e57-e60, 2011.

[15] T.-G. Lee, B. Koh, and S. Lee, "Sasang constitution as a risk factor for diabetes mellitus: a cross-sectional study," EvidenceBased Complementary and Alternative Medicine, vol. 2009, Article ID 425165, 5 pages, 2009.

[16] S. K. Lee, D. W. Yoon, J. Kim et al., "Association of adiponectin, ghrelin, and leptin with metabolic syndrome and its metabolic components in Sasang constitutional type," European Journal of Integrative Medicine, vol. 22, pp. 16-21, 2018.

[17] G. Sweeney, "Cardiovascular effects of leptin," Nature Reviews Cardiology, vol. 7, no. 1, pp. 22-29, 2010.

[18] D. D. Pham, Sasang Types may Differ in Eating Rate, Meal Size, and Regular Appetite: A Systematic Literature Review, Vol. 21, HEC Press, Montreal, Canada, 2012.

[19] M. M. Finucane, G. A. Stevens, M. J. Cowan et al., "National, regional, and global trends in body-mass index since 1980: systematic analysis of health examination surveys and epidemiological studies with 960 country-years and 9.1 million participants," The Lancet, vol. 377, no. 9765, pp. 557-567, 2011.

[20] D. Giugliano, A. Ceriello, and K. Esposito, "The effects of diet on inflammation," Journal of the American College of Cardiology, vol. 48, no. 4, pp. 677-685, 2006.

[21] J. Li, D. H. Lee, J. Hu et al., "Dietary inflammatory potential and risk of cardiovascular disease among men and women in the US," Journal of the American College of Cardiology, vol. 76, no. 19, pp. 2181-2193, 2020.

[22] A. Syauqy, C.-Y. Hsu, H.-H. Rau, and J. Chao, "Association of dietary patterns with components of metabolic syndrome and inflammation among middle-aged and older adults with metabolic syndrome in taiwan," Nutrients, vol. 10, no. 2, p. $143,2018$.

[23] J. Barbaresko, M. Koch, M. B. Schulze, and U. Nöthlings, "Dietary pattern analysis and biomarkers of low-grade inflammation: a systematic literature review," Nutrition Reviews, vol. 71, no. 8, pp. 511-527, 2013.

[24] K. M. Crowe-White, A. C. Ellis, T. Mehta, J. L. Locher, and J. D. Ard, "Dietary quality assessed by the HEI-2010 and biomarkers of cardiometabolic disease: an exploratory analysis," Journal of the American College of Nutrition, vol. 38, no. 7, pp. 640-647, 2019.

[25] Y. Baek, "Lifestyle, genomic types and non-communicable diseases in Korea: a protocol for the Korean medicine Daejeon citizen cohort study (KDCC)," BMJ Open, vol. 10, no. 4, Article ID e034499, 2020.

[26] N. C. E. Program, "Expert Panel on detection, evaluation, and treatment of high blood cholesterol in adults (adult treatment Panel III). Third report of the national cholesterol education Program (NCEP) expert panel on detection, evaluation, and treatment of high blood cholesterol in adults (adult treatment 
Panel III) final report," Circulation, vol. 106, no. 25, pp. 3143-3421, 2002.

[27] Y.-H. Baek, E.-S. Jang, K.-H. Park, J.-H. Yoo, H.-J. Jin, and S.-W. Lee, "Development and validation of brief KS-15 (Korea Sasang constitutional diagnostic questionnaire) based on body shape, temperament and symptoms," Journal of Sasang Constitutional Medicine, vol. 27, no. 2, pp. 211-221, 2015.

[28] S. J. Lee, "Biopsychological traits of Sasang typology based on Sasang personality questionnaire and body mass index," BMC Complementary and Alternative Medicine, vol. 14, no. 1, p. 315, 2014.

[29] S. W. Hong, Y. S. Suh, D. H. Kim et al., "Manifestations of sasang typology according to common chronic diseases in Koreans," Evidence-Based Complementary and Alternative Medicine, vol. 2018, Article ID 7378608, 8 pages, 2018.

[30] S. K. Chung, "Genetic loci associated with changes in lipid levels leading to constitution-based discrepancy in Koreans," BMC Complementary and Alternative Medicine, vol. 14, p. 230, 2014.

[31] K. Sun, J. Liu, and G. Ning, "Active smoking and risk of metabolic syndrome: a meta-analysis of prospective studies," PLoS One, vol. 7, no. 10, Article ID e47791, 2012.

[32] J.-h. Kang and Y.-M. Song, "Association between cotinineverified smoking status and metabolic syndrome: analyses of Korean National Health and Nutrition Examination Surveys 2008-2010," Metabolic Syndrome and Related Disorders, vol. 13, no. 3, pp. 140-148, 2015.

[33] A. Klisic, N. Kavaric, M. Jovanovic, I. Soldatovic, N. Gligorovic-Barhanovic, and J. Kotur-Stevuljevic, "Bioavailable testosterone is independently associated with Fatty Liver Index in postmenopausal women," Archives of Medical Science, vol. 5, no. 5, pp. 1188-1196, 2017.

[34] A. N. Klisic, N. D. Vasiljevic, T. P. Simic, T. I. Djukic, M. Z. Maksimovic, and M. G. Matic, "Association between C-reactive protein, anthropometric and lipid parameters among healthy normal weight and overweight postmenopausal women in Montenegro," Laboratory Medicine, vol. 45, no. 1, pp. 12-16, 2014.

[35] A. Klisic, N. Kavaric, I. Soldatovic, B. Bjelakovic, and J. KoturStevuljevic, "Relationship between cardiovascular risk score and traditional and nontraditional cardiometabolic parameters in obese adolescent girls," Journal of Medical Biochemistry, vol. 35, no. 3, pp. 282-292, 2016.

[36] Y. Yan, "Temporal relationship between inflammation and insulin resistance and their joint effect on hyperglycemia: the Bogalusa heart study," Cardiovascular Diabetology, vol. 18, no. 1, p. 109, 2019.

[37] S. Malik, N. D. Wong, S. Franklin, J. Pio, C. Fairchild, and R. Chen, "Cardiovascular disease in US Patients with metabolic syndrome, diabetes, and elevated C-reactive protein," Diabetes Care, vol. 28, no. 3, pp. 690-693, 2005.

[38] K. Yoon, S. Ryu, J. Lee, and J.-D. Park, "Higher and increased concentration of hs-CRP within normal range can predict the incidence of metabolic syndrome in healthy men," Diabetes \& Metabolic Syndrome: Clinical Research \& Reviews, vol. 12, no. 6, pp. 977-983, 2018.

[39] M.-J. Kang, "Impact of the sasang constitutional type on the high-sensitivity C-reactive protein, a predictor of cardiovascular disease," Journal of Sasang Constitutional Medicine, vol. 23, no. 4, pp. 471-486, 2011.

[40] M. D. M. Bibiloni, “Ten-year trends (1999-2010) of adherence to the mediterranean diet among the balearic islands' adult population," Nutrients, vol. 9, no. 7, p. 749, 2017.
[41] A. Sureda, M. Bibiloni, A. Julibert et al., "Adherence to the mediterranean diet and inflammatory markers," Nutrients, vol. 10, no. 1, p. 62, 2018.

[42] I. Khan, "Proinflammatory dietary intake is associated with increased risk of metabolic syndrome and its components: results from the population-based prospective study," $\mathrm{Nu}$ trients, vol. 12, no. 4, 2020.

[43] A. H. Berg and P. E. Scherer, "Adipose tissue, inflammation, and cardiovascular disease," Circulation Research, vol. 96, no. 9, pp. 939-949, 2005.

[44] C. Koebnick, M. H. Black, J. Wu et al., "A diet high in sugarsweetened beverage and low in fruits and vegetables is associated with adiposity and a pro-inflammatory adipokine profile," British Journal of Nutrition, vol. 120, no. 11, pp. 1230-1239, 2018.

[45] V. S. Malik, B. M. Popkin, G. A. Bray, J.-P. Despres, W. C. Willett, and F. B. Hu, "Sugar-sweetened beverages and risk of metabolic syndrome and type 2 diabetes: a metaanalysis," Diabetes Care, vol. 33, no. 11, pp. 2477-2483, 2010.

[46] M. C. Calle and C. J. Andersen, "Assessment of dietary patterns represents a potential, yet variable, measure of inflammatory status: a review and update," Disease Markers, vol. 2019, Article ID 3102870, 13 pages, 2019.

[47] F. Eichelmann, L. Schwingshackl, V. Fedirko, and K. Aleksandrova, "Effect of plant-based diets on obesityrelated inflammatory profiles: a systematic review and metaanalysis of intervention trials," Obesity Reviews, vol. 17, no. 11, pp. 1067-1079, 2016.

[48] M. Vitale, M. Masulli, A. A. Rivellese et al., "Influence of dietary fat and carbohydrates proportions on plasma lipids, glucose control and low-grade inflammation in patients with type 2 diabetes-The TOSCA.IT study," European Journal of Nutrition, vol. 55, no. 4, pp. 1645-1651, 2016.

[49] G. Kenđel Jovanović, I. Mrakovcic-Sutic, S. Pavičić Žeželj et al., "Metabolic and hepatic effects of energy-reduced antiinflammatory diet in younger adults with obesity," Canadian Journal of Gastroenterology and Hepatology, vol. 2021, Article ID 6649142, 12 pages, 2021.

[50] Y. Baek, "The prevalence of general and abdominal obesity according to sasang constitution in Korea," BMC Complementary and Alternative Medicine, vol. 14, no. 1, p. 298, 2014. 